\title{
Femoroacetabular impingement: prevention or intervention? The sports physician's quandary
}

\author{
J Cakic, ${ }^{1}$ J Patricios ${ }^{2,3,4}$ \\ ${ }^{1}$ The Centre for Sports Medicine and Orthopaedics, Johannesburg, South Africa; \\ ${ }^{2}$ Morningside Sports Medicine, Johannesburg, South Africa; \\ ${ }^{3}$ Section of Sports Medicine, Faculty of Health Sciences, University of Pretoria, Pretoria, South Africa; \\ ${ }^{4}$ Department of Emergency Medicine, Faculty of Health Sciences, University of the Witwatersrand, \\ Johannesburg, South Africa \\ Correspondence to Dr Jon Patricios, PO Box 1267, Parklands 2121, South Africa; jpat@mweb.co.za
}

\section{THE CONDITION}

Femoroacetabular impingement (FAl) is a mechanical process by which the human hip fails due to pathological contact between the skeletal prominences of the acetabulum and the femur. This repetitive pathological contact occurs during normal activities of daily living as well as, more prominently, in an athletic population. This causes a microtraumatic effect and subsequently irreversible chondral damage to the acetabular as well as femoral surfaces-osteoarthritis of the hip joint. ${ }^{1}$

Two types of impingement are identified: the first, pincer impingement, is due to an acetabular abnormality and results in over coverage of the femoral head. The second type, Cam impingement, results in femoral morphological change and alteration of the spherical portion at the head/neck junction of the femur. The majority of patients have a mixed picture, with features of Cam and pincer type of impingement. ${ }^{2}$

As Dr Thomas Byrd mentioned in his recent current concept ${ }^{3}$ : 'The implication of abnormal hip morphology leading to secondary joint damage had been variously described for almost a 100 years.' However, the concept of FAl as a cause of osteoarthritis is credited more recently to Ganz et al. ${ }^{1}$

\section{OCCULT SYMPTOMS...}

Typically, the patient is aware of limited hip mobility, however, symptoms of pain only appear later. Owing to this lack of pain, which would act as an 'alarm system', significant damage to the hip joint can occur before a positive impinge-ment sign (presenting in internal rotation, $90^{\circ}$ of flexion and adduction) becomes evident. It is important to emphasise that FAl is a hip disorder presenting mainly in young adults often without positive clin-ical findings. If left untreated, it can lead to significant morbidity in the form of early arthritis. Its insidious and early age of onset make FAI important in young, active sportspersons. 


\section{... WITH SIGNIFICANT SEQUELAE}

Moreover, at this age the threshold for breakdown of the chondral surface may be lower. ${ }^{4}$ In the presence of increased load and activities and vulnerable cartilage, severe damage can occur. In a less-active population, this combination of morphology and load will only present as osteoarthritis at a later stage. Early recognition and adequate treatment can improve symptoms and functionality. ${ }^{5} \mathrm{It}$ is because of this younger cohort that the need for early detection and surgical intervention has arisen.

\section{ARE THERE RISKS OF OPERATING TOO EARLY?}

Hip arthroscopy is arguably the fastest growing field in orthopaedic surgery. Patients as young as 11 years of age have been operated on. However, patient-reported outcomes following hip arthroscopy, especially where there is chondral damage, are not always favourable and may even worsen. ${ }^{6}$ Complications such as accelerated coxarthrosis and/or hip instability have occurred after acetabular rim reduction and/or excessive labral resection. ${ }^{7}$ Certainly with the high prevalence of radiographic FAI prophylactic surgery for asymptomatic FAI is inappropriate.

\section{WHAT ARE THE OTHER OPTIONS?}

FAl, similar to subacromial impingement, is defined as a decrease of clearance that results in impingement of normal daily range of motion. The early identification of those anatomical features that may pre-dispose to damage and subsequent irreversible lesions suddenly becomes appealing. Early recognition will allow us as practitioners to more effectively counsel young active patients in regard to a non-operative strategy such as modification of activity or directed physiotherapy and exercise training with the goal to prevent further progression of the condition.

Recent studies correlate radiological findings of FAI with the findings of a painful hip. Sullivan looked at 223 patients and found that $97 \%$ of patients with a painful hip had at least one abnormal finding associated with $\mathrm{FAl} ; 68 \%$ of females and $63 \%$ of males examined had two or more parameters associated with FAI. ${ }^{8}$

Yuan et al performed a study on young athletes, looking into decreased range of movements and an association with structural hip deformity, specifically in asymptomatic adolescent athletes. Eight per cent of asymptomatic teenagers had limited internal rotation $\left(<10^{\circ}\right)$ on examination; $68 \%$ of these had radiographic findings suggestive of FAI. More than two-thirds of the participants had evidence of asymptomatic hip pathological lesions on MRI. ${ }^{9}$

\section{IMPLEMENTING A SCREENING AND PREVENTATIVE INTERVENTIONAL PROGRAMME}

All around the world, clinicians are facing the same problem: how can future damage to the hip to be prevented? The South African Society of Hip Arthroscopy (SASHA) in South Africa is implementing a screening programme for teenagers in High Schools. With the cooperation of school administrators and following SASHA's Hip Prevention Examination protocol (available at http://www.sasha. org.za), SASHA clinicians plan to examine as many teenagers aged 13-14 years of age as possible, in order to isolate and 'flag' adolescents who may have hip problems in the future. Following this preventative testing, 'red-flagged' individuals will be educated and introduced to a physiotherapy-guided 
rehabilitation protocol. Parents as well as the teenagers will be given suggestions regarding their athletic involvement in school in order to protect their joints.

\section{SASHA AND SASMA}

SASHA is a small Society started in 2011 by eight enthusiastic orthopaedic surgeons who have a mutual interest in hip preservation. SASHA actively involves sports physicians and physiotherapists, has a working relationship with the South African Sports Medicine Association (SASMA), hosts workshops for SASMA members and engages therapists and rehabilitation specialists in both preventative and postoperative exercise protocols. The goal of this coordinated programme is to develop a system of recognising potential problems, developing a conservative treatment protocol and 'pre-hab' programme before making any decisions on surgical treatment. A vital component of the initiative is an educational programme targeting the medical fraternity as well as young scholars. SASHA is also involved in a surgical training programme developing an accreditation system for hip arthroscopy surgeons. The idea being that where prevention has failed, our orthopaedists will have the skills to affect a cure.

Contributors $\mathrm{JC}$ researched and drafted an original template. JP reviewed, expanded on and reformatted the article.

Competing interests None.

Provenance and peer review Not commissioned; internally peer reviewed.

\section{REFERENCES}

${ }^{1}$ Ganz R, Parvizi J, Beck M, et al. Femoroacetabular impingement: a cause for osteoarthritis of the hip. Clin Orthop Rel Res 2003;417:112-20.

${ }^{2}$ Beck M, Kalhor M, Leunig M, et al. Hip morphology influences the pattern of damage to the acetabular cartilage: femoroacetabular impingement as a cause of early osteoarthritis of the hip. J Bone Joint Surg (Br) 2005;87:1012-18.

${ }^{3}$ Byrd JWT. Femoroacetabular impingement in athletes: current concepts. Am J Sports Med 2014;42:737-51.

${ }^{4}$ Thambyah A, Broom N. How subtle structural changes associated with maturity and mild degeneration influence the impact-induced failure modes of cartilage-on-bone. Clin Biomech 2010;25:737-44.

${ }^{5}$ Beck M, Leunig M, Parvizi J, et al. Anterior femoroacetabular impingement: part II. Midterm results of surgical treatment. Clin Orthop Relat Res 2004;418:67-73.

${ }^{6}$ Kemp J, Makdissi M, Schasche A, et al. Hip chondropathy at arthroscopy: prevalence and relationship to labral pathology, femoroacetabular impingement and patient-reported outcomes. $\mathrm{Br}$ J Sports Med 2014. Published Online First: 21 March 2014. doi:10.1136/bjsports-2013-093312 
${ }^{7}$ McCarthy JC, Lee JA. Hip arthroscopy: indications, outcomes, and complications. Instr Course Lect 2006;55:301-8.

${ }^{8}$ Sullivan C, Chakraverty J, Foley K, et al. Femoroacetabular impingement: presence of FAI-like features on conventional radiography in young patients presenting to primary care with hip pain. Acta Radiolo 2013;54:690-7.

${ }^{9}$ Yuan BJ, Bartelt RB, Levy BA, et al. Decreased range of motion is associated with structural hip deformity in asymptomatic adolescent athletes. Am J Sports Med 2013;41:1519-25. 